\section{Tropical Journal of Pathology and \\ Microbiology}

2021 Volume 7 Number 2 March-April

\title{
Surrogate molecular subtyping of breast carcinomas - A study on recent modifications and their clinicopathological significance
}

\author{
Priyanka A. ${ }^{1}$, Sudalaimuthu M. ${ }^{2 *}$, Shivashekar G. ${ }^{3}$ \\ DOI: https://doi.org/10.17511/jopm.2021.i02.02 \\ 1 Priyanka A, MBBS, Department of Pathology, SRM Medical College Hospital and Research Centre, Faculty of Medicine, SRM Institute of \\ Science and Technology, Chennai, Tamilnadu, India. \\ 2* Muthu Sudalaimuthu, M.D., Department of Pathology, SRM Medical College Hospital and Research Centre, Faculty of Medicine, SRM \\ Institute of Science and Technology, Chennai, Tamilnadu, India. \\ ${ }^{3}$ G. Shivashekar, MD, DCP, Department of Pathology, SRM Medical College Hospital and Research Centre, Faculty of Medicine, SRM Institute \\ of Science and Technology, Chennai, Tamilnadu, India.
}

Context: Breast carcinoma is a heterogenous disease with varied clinicopathological features and response to therapy. Molecular classification through gene studies helps in planning therapy but has economic constraints. Hence immunohistochemical subtyping of breast carcinomas has been used as a surrogate method. Criteria for this subtyping has undergone many modifications since it was originally proposed. Objectives: To immunohistochemically subtype breast carcinomas based on St.Gallen 2017 guidelines and analyse the differences in clinicopathological parameters like age, tumour size, histopathological grade and lymph node staging between the various subtypes. Materials and methods: The study was done retrospectively at a tertiary care health centre in South India on breast carcinoma patients from January 2017 to June 2020. Immunohistochemistry was done with antibodies to the Estrogen receptor, Progesterone receptor, Human epidermal growth factor receptor-2 (HER-2) and Ki-67. Immunohistochemical Subtypes were correlated with Clinicopathological features. Results: The study had 107 cases. Hormone receptor (HR) positive HER-2 negative was the most common subtype (55 cases, $51.4 \%$ ). This subtype frequently presented without nodal metastasis (58.2\%) and in $>50$ years of age (56.4\%). Triple-negative subtype frequently presented with grade III (69.2\%), highest nodal metastasis stage (38.5\%) and in < 50 years of age (69.2\%). Conclusion: St.Gallen 2017 guidelines for immunohistochemical subtyping classified breast carcinomas into groups that differed significantly in their clinicopathological features. Further studies on differences in treatment response and survival rate differences between these different subtypes are needed.

Keywords: Breast carcinoma, Immunohistochemistry, Molecular subtyping, St. Gallen

Corresponding Author

Muthu Sudalaimuthu, M.D., Department of Pathology, SRM Medical College Hospital and Research Centre, Faculty of Medicine, SRM Institute of Science and Technology, Chennai, Tamilnadu, India.

Email: drmuthus@gmail.com
How to Cite this Article

Priyanka A, Sudalaimuthu M, Shivashekar G. Surrogate molecular subtyping of breast carcinomas - A study on recent modifications and their clinicopathological significance. Trop J Pathol Microbiol. 2021;7(2):71-77.

Available From: https://pathology.medresearch.in/ index.php/jopm/article/view/517
To Browse

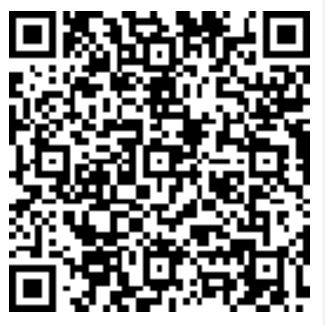

Manuscript Received 2021-03-04

Conflict of Interest No

Review Round 2
2021-04-12
Ethical Approval
Yes

Review Round 2 2021-04-08

Funding

$\mathrm{Nil}$
Review Round 3

Plagiarism X-checker $6 \%$
Accepted 2021-04-15

Note

(C) 2021 by Priyanka A, Muthu Sudalaimuthu, G. Shivashekar and Published by Siddharth Health Research and Social Welfare Society. This is an Open Access article licensed under a Creative Commons Attribution 4.0 International License https://creativecommons.org/licenses/by/4.0/ unported [CC BY 4.0]. 


\section{Introduction}

Breast cancer is one of the leading causes of cancer-related mortality and morbidity in females. Various advancements have been made in the diagnosis and treatment of breast cancer in recent decades. Due to these advancements in breast cancer therapy, the current focus is on identifying the optimal treatment strategy for individual breast cancer patients. For this purpose, breast cancer patients can be classified into various subgroups based on their clinicopathological features and the ideal treatment for each subgroup is being studied [1]. These clinicopathological variables, otherwise called the "predictive markers" indicate the sensitivity or resistance to various forms of therapies in a particular patient thereby helping to choose the ideal therapy for them.

The molecular subtype is one such predictive marker that has got prognostic significance as well [2] There are 5 molecular subtypes of breast cancer Luminal A, Luminal B, HER-2-neu enriched, Basallike and Normal breast-like. Although these subtypes indicate the response to various forms of therapies, identifying the molecular subtype in each patient needs gene assays which are costly and needs advanced equipment as well. Such facilities might not be available routinely across all the centres, particularly in the developing and underdeveloped countries.

Hence surrogate subtyping of breast cancer has been proposed based on immunohistochemical features which give results closer to the molecular subtyping. However, the criteria for this surrogate subtyping has constantly undergone modifications based on various studies. The recent 15th St. Gallen International Expert Consensus Conference on Breast Cancer held in the year 2017 has further modified the criteria for this surrogate subtyping [1]. Hence this study was done to assess the frequency of different intrinsic subtypes of breast cancer and their clinicopathological features based on these recent St. Gallen's modifications.

\section{Materials and methods}

Place of study: Our study was done at a tertiary care health centre in South India from April 2019 to June 2020.

Inclusion criteria: All cases of invasive breast carcinomas in which modified radical mastectomy had been done and received in the Department of
Pathology from January 2017 to June 2020 were included in our study.

Exclusion criteria: Post-chemotherapy patients and cases with insufficient material in blocks for immunohistochemical staining were excluded from the study. This study was approved by the Institutional Ethics Committee.

Interpretation criteria: In all these cases, specimens had been received in $10 \%$ formalin and according to the standard surgical grossing protocol appropriate bits were given. Haematoxylin and Eosin (H\&E) stained slides were examined to confirm the histological diagnosis. Histological grading was performed by Nottingham's modification of the Bloom-Richardson grading system.

Following the 8th edition of the American Joint Committee on Cancer (AJCC) cancer staging guidelines tumour size in the greatest dimension was considered. Tumours up to $20 \mathrm{~mm}$ were included under $\mathrm{T} 1,>20 \mathrm{~mm}$ up to $50 \mathrm{~mm}$ under $\mathrm{T} 2$ and $>50 \mathrm{~mm}$ under T3. Tumour of any size with direct extension to the chest wall and /or to the skin was included in T4. If no regional lymph node metastasis could be detected they were categorized as N0, metastasis in 1-3 axillary lymph nodes as $\mathrm{N} 1$, metastasis in 4-9 axillary lymph nodes as N2 and metastasis in $>10$ axillary lymph nodes as N3.

For the immunohistochemistry (IHC) procedure, the peroxidase anti peroxidase (PAP) method was followed. Representative blocks were submitted for IHC after screening all sections. 3-5 micron thickness sections were taken on positively charged slides and incubated. Slides were deparaffinised and dehydrated in xylene, absolute alcohol. The antigen retrieval process was done in microwave cooking with Tris-EDTA (ethylenediaminetetraacetic acid) buffer solution maintained under $\mathrm{pH} 9$.

Then sections were treated with 3\% hydrogen peroxide. Sections were stained with primary antibody for 1 hour. Antibodies to estrogen receptor $(E R)$, progesterone receptor (PR), human epidermal growth factor receptor- 2 (HER-2) were used. All were monoclonal rabbit antibodies (PathnSitu). Then polyexcel horseradish peroxidase (HRP) labelled secondary antibody was added and incubated. Then 3, 3'diaminobenzidine (DAB) chromogen was added and counterstained with Harris haematoxylin for 30 seconds.

As per the American Society of Clinical Oncology/ 
College of American Pathologists (ASCO/CAP) guidelines ER/PR were considered positive when $1 \%$ or more cells showed nuclear staining. For HER -2 , if there were no membranous staining or faint incomplete membranous staining in less than $10 \%$ of cells, the score was given as zero.

Faint incomplete membranous staining in $>10 \%$ of cells was scored $1+$. Weak to moderate complete membranous staining in $>10 \%$ cells was scored $2+$. Strong complete membranous staining in $>10 \%$ cells was given a score of $3+$. Cases with score 0 and 1 were considered HER-2 negative. Score 3+ was taken as HER- 2 positive. Score 2 was taken as an equivocal result and Fluorescence in situ hybridization (FISH) was done in these cases to confirm HER-2 status.

Based on the expression of ER, PR and HER-2, all the cases were subtyped into four categories according to St. Gallen 2017 guidelines. These four categories were triple-negative (hormone receptor (HR) negative, HER-2 negative) tumours, HR negative HER-2-positive tumours, HR-positive HER-2 positive tumours and HR-positive HER-2 negative tumours. The last group was further classified into "Luminal A-like" (low grade) and "Luminal B-like" (high grade) tumours.

For grade II cases, Ki-67 immunohistochemical staining (PathnSitu) was done and when $<20 \%$ cells were positive they were categorised as "Luminal Alike" tumours. When $>20 \%$ cells were Ki-67 positive they were categorised as "Luminal B- like tumours". Clinicopathological parameters like age, $\mathrm{T}$ (tumour size) staging, N (lymph node) staging and histopathological grading were studied in all these groups and compared.

Statistical analysis: Statistical analysis of the data was done using SPSS software 24.0 by Chi-square test. p-value less than 0.05 was considered statistically significant.

\section{Results}

Our study had 107 cases based on the inclusion and exclusion criteria. HR-positive HER-2 negative subtype (55 cases, $51.4 \%$ ) was the most common subtype in our study with 38 "luminal Alike" cases ( $35.5 \%$ ) and 17 "luminal B- like" cases ( 15.9\%). This was followed by HR negative HER-2 positive and triple-negative subtypes (Table I). HR-positive HER-2 positive subtype was the least common subtype in our study with 11 cases $(10.3 \%)$. These subtypes showed significant differences in their clinicopathological features.

Triple-negative subtype cases more often presented with grade III ( $69.2 \%)$. HR-positive HER-2 positive $(81.8 \%)$ cases more often presented with grade II. Triple-negative, HR-positive HER-2 positive subtypes never presented with grade I (Table II). On the other hand Grade I tumours were more common in HR-positive HER-2 negative subtype when compared with other subtypes. The association between various subtypes and grade was found to be statistically significant ( $p$-value $<0.001$ ).

Triple-negative subtype cases also frequently had the highest stage of lymph node metastasis (N338.4\%), whereas HR-positive HER-2 negative subtype often did not show nodal metastasis (NO$58.2 \%$ )(Table III). In the latter subtype, "luminal Alike" cases often did not show nodal metastasis (76.3\%) compared to "luminal B -like cases $(17.6 \%)$ The association between various subtypes and lymph node staging was found to be statistically significant ( $p$-value $<0.001$ ).

TABLE I: Immunohistochemical subtypes of breast carcinoma in our study

\begin{tabular}{|l|l|}
\hline Immunohistochemical subtypes & Number of cases $(\%) ; \mathbf{n = 1 0 7}$ \\
\hline Triple negative & $13(12.1 \%)$ \\
\hline HR -,HER-2 + & $28(26.2 \%)$ \\
\hline HR+, HER-2 + & $11(10.3 \%)$ \\
\hline HR+,HER-2 - & $55(51.4 \%)$ \\
\hline
\end{tabular}

(HR- hormone receptor; HER-2 -human epidermal growth factor receptor -2)

TABLE II: Histological grade in different immunohistochemical subtypes of breast carcinoma

\begin{tabular}{|l|l|l|l|l|l|}
\hline \multicolumn{1}{|c|}{ Immunohistochemical subtypes } & \multicolumn{1}{|c|}{ Grade I } & \multicolumn{1}{c|}{ Grade II } & \multicolumn{1}{c|}{ Grade III } & \multicolumn{1}{c|}{ X2 - Value } & \multicolumn{1}{c|}{-Value } \\
\hline Triple Negative $(n=13)$ & $0(0.0 \%)$ & $4(30.8 \%)$ & $9(69.2 \%)$ & 27.087 & $<0.001$ \\
\hline HR-,HER-2+ $(n=28)$ & $2(7.1 \%)$ & $19(67.9 \%)$ & $7(25 \%)$ & & \\
\hline HR+,HER-2+ $(n=11)$ & $0(0.0 \%)$ & $9(81.8 \%)$ & $2(18.2 \%)$ & & \\
\hline HR+,HER-2- $(n=55)$ & $15(27.3 \%)$ & $36(65.4 \%)$ & $4(7.3 \%)$ & & \\
\hline
\end{tabular}

(HR- hormone receptor; HER-2 -human epidermal growth factor receptor -2) 
TABLE III: Lymph node staging in different immunohistochemical subtypes ( $\mathrm{N}$ stage)

\begin{tabular}{|l|l|l|l|l|l|l|}
\hline \multicolumn{1}{|c|}{ Immunohistochemical subtypes } & \multicolumn{1}{|c|}{ N0 } & \multicolumn{1}{|c|}{ N1 } & \multicolumn{1}{|c|}{ N2 } & \multicolumn{1}{c|}{ N3 } & \multicolumn{1}{c|}{ X2 -Value } & \multicolumn{1}{c|}{ p-Value } \\
\hline Triple Negative $(n=13)$ & $2(15.4 \%)$ & $2(15.4 \%)$ & $4(30.8 \%)$ & $5(38.4 \%)$ & 54.528 & $<0.001$ \\
\hline HR- , HER-2+ $(n=28)$ & $4(14.3 \%)$ & $5(17.8 \%)$ & $19(67.9 \%)$ & $0(0.0 \%)$ & \\
\hline HR+, HER-2+ $(n=11)$ & $4(36.4 \%)$ & $5(45.4 \%)$ & $0(0.0 \%)$ & $2(18.2 \%)$ & & \\
\hline HR+, HER-2- $(n=55)$ & $32(58.2 \%)$ & $17(30.9 \%)$ & $4(7.3 \%)$ & $2(3.6 \%)$ & & \\
\hline
\end{tabular}

(HR- hormone receptor; HER-2 -human epidermal growth factor receptor -2)

TABLE IV: Tumour staging in different immunohistochemical subtypes (T stage)

\begin{tabular}{|l|l|l|l|l|l|l|}
\hline \multicolumn{1}{|c|}{ Immunohistochemical subtypes } & \multicolumn{1}{|c|}{ T1 } & \multicolumn{1}{|c|}{ T2 } & \multicolumn{1}{|c|}{ T3 } & \multicolumn{1}{c|}{ T4 } & \multicolumn{1}{c|}{ X2 -Value } & \multicolumn{1}{c|}{ p-Value } \\
\hline Triple Negative $(n=13)$ & $0(0.0 \%)$ & $8(61.5 \%)$ & $4(30.8 \%)$ & $1(7.7 \%)$ & 31.395 & $<0.001$ \\
\hline HR- ,HER-2+ $(n=28)$ & $0(0.0 \%)$ & $20(71.4 \%)$ & $8(28.6 \%)$ & $0(0.0 \%)$ & & \\
\hline HR+,HER-2+ $(n=11)$ & $2(18.2 \%)$ & $8(72.7 \%)$ & $0(0.0 \%)$ & $1(9.1 \%)$ & & \\
\hline HR+,HER-2- $(n=55)$ & $16(29.1 \%)$ & $37(67.3 \%)$ & $2(3.6 \%)$ & $0(0.0 \%)$ & & \\
\hline
\end{tabular}

(HR- hormone receptor; HER-2 -human epidermal growth factor receptor -2)

\section{TABLE V: Immunohistochemical subtypes and Age}

\begin{tabular}{|l|l|l|l|l|}
\hline \multicolumn{1}{|c|}{ Immunohistochemical subtypes } & \multicolumn{1}{|c|}{ Below 50 years } & \multicolumn{1}{c|}{ Above 50 years } & \multicolumn{1}{c|}{ X2 -Value } & \multicolumn{1}{c|}{$\mathbf{p}$-Value } \\
\hline Triple Negative $(n=13)$ & $9(69.2 \%)$ & $4(30.8 \%)$ & 3.001 & 0.401 \\
\hline HR- ,HER-2+ $(n=28)$ & $12(42.9 \%)$ & $16(57.1 \%)$ & & \\
\hline HR+,HER-2+ $(n=11)$ & $5(45.5 \%)$ & $6(54.5 \%)$ & & \\
\hline HR+,HER-2- $(n=55)$ & $24(43.6 \%)$ & $31(56.4 \%)$ & & \\
\hline
\end{tabular}

(HR- hormone receptor; HER-2 -human epidermal growth factor receptor -2)

Percentage of larger tumours (T3) was maximum in the triple-negative subtype $(30.8 \%)$ and $H R$ negative HER-2 positive subtype (28.6\%) when compared to other subtypes (Table IV). On the other hand, smaller tumours (T1) were more frequent in the HR-positive HER-2 negative group (29.1\%).

In this subtype also smaller tumours were more common in "luminal Alike" cases (42.1\%) compared to "luminal B- like " cases. Triple-negative and HR negative HER -2 positive rarely presented with $\mathrm{T} 1$ tumours. The association between various subtypes and tumour size was also found to be statistically significant ( $p$-value $<0.001$ ).

With regards to age, triple-negative (69.2\%) subtype often presented in women of the age group of fewer than 50 years (Table V). All the other subtypes were more common in women of more than 50 years. On the statistical analysis of data, the association between various subtypes and age was not significant ( $p$ value $=0.401$ )

\section{Discussion}

Breast cancer is a heterogeneous disease with varied clinicopathological features. As a result all the cases do not respond uniformly to the different forms of therapy.
Molecular classification of breast carcinomas through gene profiling studies is a useful tool in planning therapy for breast cancer patients as some subtypes respond well to endocrine therapy whereas others respond better to chemotherapy. But due to the lack of facilities and increased expenditure it cannot be used in day to day practice in all economic setups. Therefore immunohistochemistry based subtyping of breast carcinomas was suggested as a surrogate method and is being widely used [3]. This subtyping of breast cancer based on immunohistochemistry has been constantly under evolution with repeated modifications in criteria based on new studies, advancements and practical experience. The St. Gallen's Guidelines for immunohistochemical subtyping of breast carcinomas which has been accepted by the World Health Organization was first suggested in 2011 [4] and has undergone various modifications in the subsequent St.Gallen conferences. This study was based on the recent 2017 guidelines [1] which had recommended that molecular subtype terms should not be used in the surrogate immunohistochemical subtyping as the results differ in some cases. However the guidelines recommended that immunohistochemical subtyping of breast carcinomas should be continued but with certain modifications in the types, terminology and criteria. 
They further recommended this classification to be used in planning treatment strategy in individual breast carcinoma patients. HR-positive HER-2 negative was the most common subtype in our study. This in turn was due to the high frequency of "luminal Alike" cases. In the earlier classificatio, " luminal $A$ " used to be a separate subtype and was the most common subtype in many previous studies $[3,5,6,7,8,9]$. Studies on the Indian population done by Munjal et al [10] and Ashima et al [9] had also shown "luminal Alike" as the predominant subtype. Howeve few other Indian studies by Shabnam et al [11] and Kunheri et al [12] observed triple-negative and "luminal B -like" to be the most common subtypes respectively. It needs to be studied further whether this is a geographic variation in different parts of India due to some genetic and geographic factors.

Subtypes and tumour size: Our study found a significant association between the subtypes and the tumour size. The The proportion of tumours larger than $5 \mathrm{cms}$ was maximum intriple-negative subtype in our study. Spitale et al [3] had observed the same in their study. After thetriple-negative subtype, the frequency of such larger tumours was maximum in the HR negative HER-2 positive subtype. Studies by Onitillo et al [13] and Engstrom et al [14] had also observed that tumours larger than $5 \mathrm{~cm}$ were frequent in this subtype. At the other end, tumours less than $2 \mathrm{cms}$ were frequent in theHR-positive HER-2 negative subtype particularly in the "Luminal Alike" cases in our study. Such an association between small tumour size and "Luminal Alike" cases was noted in many previous studies as well $[3,5,7,13,14]$. Although few studies have noted no significant association between subtypes and tumour size they seem infrequent $[10,15]$.

Subtypes and lymph node metastasis: The The triple-negative type was frequently associated with the the highest stage of lymph node metastasis in our study. In contrast to our results nodal metastasis was frequently absent in triple-negative subtype in studies by Spitale et al and Vasconcelos et al $[3,5]$. However many other study results by Zaha et al, Munjal et al, Kunheri et al and Blows et al were similar to our study strengthening our results $[6,10,12,16]$. Lymph node metastasis was often absent in "Luminal Alike" tumours in our study. This was similar to the results of Tang et al [17] and Onitillo et al [13].

Subtypes and tumour grade: Triple-negative subtype cases were often of the highest histological
Grade in our study similar to previous studies [3, $5,7,12,15]$. "Luminal Alike" cases showed the the maximum percentage of Grade I tumours. These findings were also consistent with previous $[3,5,8,10,13,14,15]$ studies. Statistically significant difference was noted in the histological grade between various subtypes in our study, similar to many previous studies $[3,6,7,10,15,16,18,19]$.

Subtypes and age of the patient: Although our data showed no significant difference in the age of presentation in various subtypes $p$-value $=0.401$ ), our results were comparable to previous studies. Triple-negative subtype involved a younger age group in our study, similar to studies by Spitale et $a$, Vasconcelos et al and Yinghao et al $[3,5,8]$. "LuminaAlike" cases presented in an older age group in our study, a finding that was noted in Spitale et al [3] and Yinghao et al [8] studies as well. Thus although statistical significance was not reached, our results were in line with the previous studies.

New entities in recent classification: HR-positive HER-2 positive andHR-positive HER-2 negative were two new entities proposed only in the latest St. Gallen guidelines. Hence we could not compare our results in these groups with many previous studies. HR-positive HER-2 positive subtype frequently presented in more than 50 years women with T2 size and grade II tumours. HR-positive HER-2 negative subtype also frequently presented with similar features but with less frequent nodal metastasis. Although some of these findings were similar to the study by Cheng et al [20], we could not compare our results with most studies since these two are new entitiesthat came only after the new classification. Being new entitie, more studies need to be done which might confirm our finding.

Significance of "luminal Alike "and " luminal Blike" tumours: One of the major grey zones in the immunohistochemical subtyping of breast carcinomas is in distinguishing the "Luminal A- like" and "Luminal B- like" tumours among the HRpositive HER-2 negative cases. In fact this was the major area of a dilemma for so many years and so many modifications were repeatedly being done in the criteria to distinguish these two entities. When we classified these tumours based on the recent St. Gallen guidelines [1], Cases in the resulting two groups differed significantly in their clinicopathological features reiterating the validity of these guidelines. 
While "Luminal A- like" tumours were often of smaller tumour size without nodal metastasis in many cases, "Luminal-B" like tumours were often of a larger tumours size with nodal metastasis.

One of the limitations of our study was we were not able to do a long term follow up of these patients to assess the difference in treatment response and survival rates among the different subtypes. This was because many patients were lost to follow-up. Another limitation was $\mathrm{Ki}-67$ was not done in all cases due to economic constraints.

However this was not necessary in all cases as according to the St. Gallen guidelines, the grade of the tumour can be used instead of $\mathrm{Ki}-67$ for classifying the "Luminal A- like" and "Luminal Blike" tumours. We followed the same guidelines. In cases where the grade was intermediate alone, we used Ki-67 for classification. Such an approach would be cost-effective for subtyping breast carcinomas in developing countries.

\section{Conclusion}

Breast cancer is a heterogeneous disease with different subtypes differing in their clinicopathological features. Since these subtypes are likely to differ in their treatment response as well, it is essential to identify the subtype in every individual patient.

Since molecular subtyping might not be affordable in developing and undeveloped countries, surrogate immunohistochemical subtyping can be used. We adopted the recent St. Gallen guidelines for this immunohistochemical subtyping and observed that the subtypes differed significantly in their clinicopathological features further validating the guidelines.

Further long term studies with a large sample size and adequate follow up will help in assessing the treatment response and the survival rates in these different subtypes. Such large scale studies will help in planning the treatment guidelines for each subtype particularly in the Indian population.

\section{Acknowledgement}

We thank all our Department faculties, Postgraduates and Technicians for their kind help during the study. I thank Mrs. Anitha (statistician) for helping me in statistical analysis.

\section{Reference}

01. Curigliano G, Burstein HJ, P Winer E, Gnant $M$, Dubsky $P$, Loibl $S$, Colleoni $M$, Regan MM, Piccart-Gebhart M, Senn HJ, Thürlimann B. Deescalating and escalating treatments for earlystage breast cancer- the St Gallen International Expert Consensus Conference on the Primary Therapy of Early Breast Cancer 2017. Annals of Oncology. 2017 Aug $1 ; 28(8) 1700-12$.

[Crossref]

02. Eliyatkın N, Yalçın E, Zengel B, Aktaş S, Vardar E. Molecular classification of breast carcinomafrom traditional, old-fashioned way to a new age, and a new way. The journal of breast health. 2015 Apr;11(2)59.

[Crossref]

03. Spitale A, Mazzola P, Soldini D, Mazzucchelli L, Bordoni A. Breast cancer classification according to immunohistochemical markersclinicopathologic features and short-term survival analysis in a population-based study from the South of Switzerland. Annals of oncology. 2009 Apr 1;20(4)628-35.

[Crossref]

04. Gnant M, Harbeck N, Thomssen C. St Gallen 2011- summary of the consensus discussion. Breast care. 2011;6(2)136-41.

[Crossref]

05. Vasconcelos I, Hussainzada A, Berger S, Fietze E, Linke J, Siedentopf F, Schoenegg W. The St Gallen surrogate classification for breast cancer subtypes successfully predicts tumor presenting features, nodal involvement, recurrence patterns and disease free survival. The Breast. 2016 Oct $1 ; 29 ; 181-5$.

[Crossref]

06. Zaha DC, Lazar E, Lazureanu C. Clinicopathologic features and five years survival analysis in molecular subtypes of breast cancer. Rom J Morphol Embryol. 2010 Jan $1 ; 51(1) 85$.

[Crossref]

07. Yang $X R$, Sherman ME, Rimm DL, Lissowska J, Brinton LA, Peplonska B, Hewitt SM, Anderson WF, Szeszenia-Dąbrowska N, Bardin-Mikolajczak A, Zatonski W. Differences in risk factors for breast cancer molecular subtypes in a population-based study. Cancer Epidemiology and Prevention Biomarkers. 2007;Mar-1;16(3) 439-43. [Crossref] 
08. Su Y, Zheng Y, Zheng W, Gu K, Chen Z, Li G, Cai $\mathrm{Q}$, Lu W, Shu XO. Distinct distribution and prognostic significance of molecular subtypes of breast cancer in Chinese women- a populationbased cohort study. BMC cancer. 2011 Dec $1 ; 11(1) 292$.

[Crossref]

09. Batra A, Marwah N, Marwah S, Gupta S, Dharembra D, Sen R. St Gallen's molecular subtypes in primary breast carcinoma in Indian population. Clinical Cancer Investigation Journal. 2016 Sep 1;5(5)416.

[Crossref]

10. Munjal K, Ambaye A, Evans MF, Mitchell J, Nandedkar S, Cooper K. Immunohistochemical analysis of $E R, P R$, Her2 and CK5/6 in infiltrative breast carcinomas in Indian patients. Asian Pac J Cancer Prev. 2009 Jan 1;10(5)773-8. [Crossref]

11. Karangadan S, Patil AG, Andola SK. Immunohistochemical characterization of molecular classification of breast carcinoma and its relation with $\mathrm{Ki}-67$. Clinical Cancer Investigation Journal. 2016 Sep 1;5(5)430. [Crossref]

12. Kunheri B, Raj RV, Vijaykumar DK, Pavithran K. Impact of St Gallen surrogate classification for intrinsic breast cancer sub-types on disease features, recurrence, and survival in South Indian patients. Indian Journal of Cancer. 2020 Jan $1 ; 57(1) 49$.

[Crossref]

13. Onitilo AA, Engel JM, Greenlee RT, Mukesh BN. Breast cancer subtypes based on ER/PR and Her2 expression- comparison of clinicopathologic features and survival. Clinical medicine \& research. 2009 Jun 1;7(1-2)4-13. [Crossref]

14. Engstrøm MJ, Opdahl S, Hagen AI, Romundstad PR, Akslen LA, Haugen OA, Vatten LJ, Bofin AM. Molecular subtypes, histopathological grade and survival in a historic cohort of breast cancer patients. Breast cancer research and treatment. 2013 Aug 1;140(3)463-73.

[Crossref]
15. Piñero-Madrona A, Polo-García L, AlonsoRomero JL, Salinas-Ramos J, Canteras-Jordana M, Sola-Pérez J, Galindo-Fernández PJ, IllanaMoreno J, Bermejo-López J, Navarrete-Montoya A, Parrilla-Paricio P. Immunohistochemical characterisation of breast cancer- towards a new clasification?. Cirugia espanola. 2008 Sep $1 ; 84(3) 138-45$.

[Crossref]

16. Blows FM, Driver KE, Schmidt MK, Broeks A, Van Leeuwen FE, Wesseling J, Cheang MC, Gelmon K, Nielsen TO, Blomqvist C, Heikkilä P. Subtyping of breast cancer by immunohistochemistry to investigate a relationship between subtype and short and long term survival- a collaborative analysis of data for 10,159 cases from 12 studies. PLoS med. 2010 May 25;7(5)e1000279.

[Crossref]

17. Tang $P$, Skinner KA, Hicks DG. Molecular classification of breast carcinomas by immunohistochemical analysis- are we ready?. Diagnostic Molecular Pathology. 2009 Sep $1 ; 18(3) 125-32$.

[Crossref]

18. Carey LA, Perou CM, Livasy CA, Dressler LG, Cowan D, Conway K, Karaca G, Troester MA, Tse $\mathrm{CK}$, Edmiston S, Deming SL. Race, breast cancer subtypes, and survival in the Carolina Breast Cancer Study. Jama. 2006 Jun 7;295(21)2492-502.

[Crossref]

19. Cheang MC, Chia SK, Voduc D, Gao D, Leung S, Snider J, Watson M, Davies S, Bernard PS, Parker JS, Perou CM. Ki67 index, HER2 status, and prognosis of patients with luminal $\mathrm{B}$ breast cancer. JNCI- Journal of the National Cancer Institute. 2009 May 20;101(10)736-50.

[Crossref]

20. Cheang MC, Voduc D, Bajdik C, Leung S, McKinney S, Chia SK, Perou CM, Nielsen TO. Basal-like breast cancer defined by five biomarkers has superior prognostic value than triple-negative phenotype. Clinical cancer research. 2008 Mar 1;14(5)1368-76.

[Crossref] 